\title{
PLANETARY NEBULAE OF TYPE I REVISITED
}

\author{
S. TORRES-PEIMBERT AND M. PEIMBERT \\ Instituto de Astronomía, UNAM \\ Apartado Postal 70-264, México 04510, D. F., México
}

\begin{abstract}
.
Type I PNe were defined in 1977; during the last 19 years evidence has accumulated indicating that indeed these objects form a distinct physical group, corresponding to the high mass end of the stellar progenitors of $\mathrm{PNe}$. In this review the definition of PNe of Type I is reanalyzed. Their properties in our Galaxy and other galaxies are discussed.
\end{abstract}

\section{Introduction}

Peimbert (1978) defined He-N rich objects as PNe of Type I. Based on their kinematic properties and galactic distribution he suggested that the masses of the progenitor stars were higher than those of other PNe. Peimbert \& Torres-Peimbert (1983), presented a list of $29 \mathrm{PNe}$ of Type I and pointed out that most of them were bipolar. Peimbert (1990) reviewed some results derived from the study of Type I PNe.

By combining results from $\mathrm{PNe}$ in the Galaxy and in the Magellanic Clouds, MC, it has been possible to advance considerably in the understanding of Type I PNe. Observations of MC PNe permit us to determine with high accuracy the ionized mass of their envelopes and the mass of their central stars because their distances are well known. Alternatively observations of galactic PNe permit us to do detailed studies of single objects, including their internal velocity structure and spatial distribution of their molecular component, while from the dynamical properties and distribution of a large sample of objects it is possible to estimate the average main sequence mass of their progenitors. 


\section{S. Torres-Peimbert}

\section{Type I PNe as a Physical Group}

There are at least eight properties that are common to Type I PNe and that, in general, are not present in other types of $\mathrm{PNe}: \mathrm{a}$ ) high $\mathrm{He}$ and $\mathrm{N}$ abundances, b) bipolar structure, c) high central star mass (often combined with high central stellar temperature), d) high envelope mass, e) high main sequence mass of the progenitor star, $\mathrm{f}$ ) presence of $\mathrm{H}_{2}$ collisionally excited, and $\mathrm{CO}, \mathrm{g}$ ) complex velocity field including high velocities (although not all the $\mathrm{PNe}$ with high velocities are of Type I), and $\mathrm{h}$ ) large differences between $T\left(\mathrm{O}^{++}\right)$and $T\left(\mathrm{C}^{++}\right)$. Different authors have found strong correlations between two or more of the properties mentioned above.

There are three morphological classifications that have been widely used. Greig $(1971,1972)$ divided PNe into four main classes: A (annular), B (binebulous), $\mathrm{C}$ (centric, surface density increases toward the center), and E (egg shaped). Balick $(1987,1989)$ divided PNe into three classes: round, elliptical and butterfly. Stanghellini et al. (1993) divided PNe into four classes: irregular, pointsymmetrical, bipolar and elliptical. Objects with a bipolar structure have been called B, bipolar, binebulous, hourglass and butterfly by different authors, we will call them bipolar objects.

Most galactic He- $\mathrm{N}$ rich $\mathrm{PNe}$ are bipolar and most bipolar PNe are $\mathrm{He}-\mathrm{N}$ rich (e. g., Peimbert \& Torres-Peimbert 1983; Gorny et al. 1996). Dopita et al. (1996) have found from HST observations that the He-N rich PNe in the $\mathrm{MC}$ are bipolar; this result is very important because for galaxies farther away than the $\mathrm{MC}$ it is possible to determine the $\mathrm{He}$ and $\mathrm{N}$ abundances of $\mathrm{PNe}$ but not their morphology.

In general, central stars of $\mathrm{He}-\mathrm{N}$ rich $\mathrm{PNe}$ have higher masses than the central stars of other PNe (e. g., Kaler 1983; Gathier \& Pottasch 1985; Kaler \& Jacoby 1989, 1990; Pottasch 1989; Kaler et al. 1990; Stasinska \& Tylenda 1990; Dopita 1993; Jacoby \& Kaler 1993; Cazetta \& Maciel 1994; Gorny et al. 1996).

Walton et al. (1991) found for 35 non Type I PNe in the MC a mean mass $\left\langle\mathrm{M}\left(\mathrm{H}^{+}\right)\right\rangle=(0.217 \pm 0.079) \mathrm{M}_{\odot}$, for 3 Type I objects in the SMC $\left\langle\mathrm{M}\left(\mathrm{H}^{+}\right)\right\rangle=(0.28 \pm 0.06) \mathrm{M}_{\odot}$ and for 7 Type I PNe in the LMC $\left\langle\mathrm{M}\left(\mathrm{H}^{+}\right)\right\rangle$ $=(0.36 \pm 0.09) \mathrm{M}_{\odot}$. To obtain the total $\mathrm{H}$ mass of the envelopes it is necessary to estimate the amount of neutral and molecular H. Sabbadin (1986) finds that B nebulae spend most of their life as optically thick, whereas $\mathrm{C}$ nebulae spend most of their life as optically thin; this result implies that the amount of $\mathrm{H} \mathrm{I}$ and $\mathrm{H}_{2}$ in Type I PNe is considerably larger than in non Type I PNe (see also Kastner et al. 1996). Gómez et al. (1989) find for NGC 6302, a PN of Type I, that $\mathrm{M}\left(\mathrm{H}^{+}\right) \sim 0.20 \mathrm{M}_{\odot}$, $\mathrm{M}(\mathrm{H} \mathrm{I}) \sim 0.05 \mathrm{M}_{\odot}$ and $\mathrm{M}\left(\mathrm{H}_{2}\right) \sim 0.5 \mathrm{M}_{\odot}$. Therefore from the previous considerations it follows that the results from the MC imply that the shell 


\section{PLANETARY NEBULAE OF TYPE I REVISITED}

masses of Type I PNe are considerably larger than those of non Type I objects.

Different kinematical and galactic distribution studies have shown that bipolar and Type I PNe as a group correspond to more massive stellar progenitors than the other types of PNe (Greig 1972; Cudworth 1974; Corradi \& Schwarz 1995; Kastner et al. 1996; Gorny et al. 1996; Acker 1980, 1983; Dutra \& Maciel 1990; Maciel \& Dutra 1992; Pasquali \& Perinotto 1993).

Webster et al. (1988), Zuckerman \& Gatley (1988), Huggins (1993) and Kastner et al. (1996), have found that most of the objects with $\mathrm{H}_{2}$ lines in emission show a bipolar structure, and that the emission originates in the disk that surrounds the central star. Huggins et al. (1996) from CO observations, have found a strong correlation between the molecular mass, bipolarity, and $\mathrm{N} / \mathrm{O}$.

By combining the C III] $1906+1909$ with the C II 4267 and the [O III] $4363 / 5007$ line intensities it is possible to determine $T\left(\mathrm{C}^{++}\right)$and $T\left(\mathrm{O}^{++}\right)$ respectively. Based on a compilation of line intensities by Rola \& Stasinska (1994) Peimbert et al. (1995b) determined $T\left(\mathrm{C}^{++}\right)$and $T\left(\mathrm{O}^{++}\right)$for $36 \mathrm{PNe}$, including a total of 68 independent positions; they find that, in general, $T\left(\mathrm{O}^{++}\right)>T\left(\mathrm{C}^{++}\right)$and that the mean value is $\left\langle T\left(\mathrm{O}^{++}\right)-T\left(\mathrm{C}^{++}\right)\right\rangle=$ $1480 \mathrm{~K}$. Type I PNe show typical differences of about $3000 \mathrm{~K}$; for $\mathrm{Hu} 1-2$, a Type I PNe, the difference reaches $6500 \mathrm{~K}$. The objects with largest $T\left(\mathrm{O}^{++}\right)$ $-T\left(\mathrm{C}^{++}\right)$values are those that show complex gas motions, that often reach velocities higher than $100 \mathrm{~km} \mathrm{~s}^{-1}$. The complex velocity fields support the idea that shocks are responsible for most of the $T\left(\mathrm{O}^{++}\right)-T\left(\mathrm{C}^{++}\right)$ difference; additional support for this idea comes from the extensive grid of shock models by Dopita \& Sutherland (1996) where it is found that shock waves produce considerably higher $T\left(\mathrm{O}^{++}\right)$than $T\left(\mathrm{C}^{++}\right)$values, while $T\left(\mathrm{O}^{++}\right)$and $T\left(\mathrm{C}^{++}\right)$are practically the same for the photoionized precursors.

There are other non bipolar PNe that show high velocities and evidence for shock activity. NGC 2392, the Eskimo Nebula, shows velocities as high as $190 \mathrm{~km} \mathrm{~s}^{-1}$, but it is not He-N rich. Similarly NGC 7009, a point symmetric nebula, shows large $T\left(\mathrm{O}^{++}\right)-T\left(\mathrm{C}^{++}\right)$values and is not He-N rich. Gorny et al. (1996) find that point symmetric PNe form a distinct physical group with $\mathrm{N} / \mathrm{O}$ values smaller and distances to the galactic plane higher than those of bipolar nebulae, indicating that their progenitor stars have smaller masses than those of bipolar nebulae. 


\section{S. Torres-Peimbert}

\section{Definitions}

Type I PNe were defined as having $\mathrm{He} / \mathrm{H}>0.125$ or $\mathrm{N} / \mathrm{O}>0.5$ (Peimbert 1978, 1985, 1990; Peimbert \& Torres-Peimbert 1983; Calvet \& Peimbert 1983). Kingsburgh \& Barlow (1994) defined Type I PNe as having a N/O ratio higher than their initial $(\mathrm{C}+\mathrm{N}) / \mathrm{O}$ ratio, implying a complete conversion of the initial $\mathrm{C}$ into $\mathrm{N}$ and that part of the $\mathrm{C}$ produced by the third dredge-up was converted into $\mathrm{N}$; based on the solar neighborhood $\mathrm{H}$ II regions they defined as Type I PNe those with $\mathrm{N} / \mathrm{O}>0.8$. There are two minor problems with the adopted numerical ratio: a) the interstellar $\mathrm{C} / \mathrm{O}$ ratio has been increasing with time and the $\mathrm{C} / \mathrm{O}$ ratio with which the progenitors of $\mathrm{PNe}$ formed was smaller than the present $\mathrm{C} / \mathrm{O}$ ratio (e. g., Carigi 1994; Garnett et al. 1995), b) the presence of the $\mathrm{O}^{++}+\mathrm{H}^{0} \rightarrow \mathrm{O}^{+}$ $+\mathrm{H}^{+}$charge exchange reaction makes the derived $\mathrm{N} / \mathrm{O}$ ratio based on the $\mathrm{N}^{+} / \mathrm{O}^{+}$ratio a lower limit to the real value (see below).

The ISM abundances in the MC and the Galaxy are different, therefore the definitions have to take these differences into account. From the $\mathrm{H}, \mathrm{He}$, $\mathrm{C}, \mathrm{N}$, and $\mathrm{O}$ abundances in the MC (Dufour 1984, and references therein; Garnett et al. 1995), the adopted $\mathrm{He} / \mathrm{H}$ value in the Galaxy for Type I PNe and the suggestion by Kingsburgh \& Barlow (1994) on the N/O ratio, we propose the following definitions for Type I PNe: for the LMC, He/H $>0.105$ or $\mathrm{N} / \mathrm{O}>0.38$ and for the $\mathrm{SMC} \mathrm{He} / \mathrm{H}>0.100$ or $\mathrm{N} / \mathrm{O}>0.22$. For the reasons mentioned in the previous paragraph the N/O limits could be somewhat smaller. For the MC Type I PNe Dopita \& Meatheringham (1991) have adopted N/O $>0.30$, and Leisy \& Dennefeld $\mathrm{He} / \mathrm{H}>0.10$ and $\mathrm{N} / \mathrm{O}>0.25$, in excellent agreement with the previous definitions. Dopita \& Meatheringham find some objects with appreciable He enhancements without any corresponding $\mathrm{N} / \mathrm{O}$ enhancement, a signature of the third dredge up phase without $\mathrm{C} / \mathrm{N}$ conversion.

\section{Abundances}

Errors in the abundance determinations of $\mathrm{He} / \mathrm{H}$ and $\mathrm{N} / \mathrm{O}$ can produce errors in the classification of $\mathrm{PNe}$. In particular collisional excitation of the He I lines is very efficient in objects with high temperatures and high densities, like those of $\mathrm{MC} \mathrm{PNe}$, and if it is not taken into account the $\mathrm{He} / \mathrm{H}$ value would be overestimated. For example the $\mathrm{He} / \mathrm{H}$ value derived for the LMC Type I PN N102 by Dopita \& Meatheringham (1991), without taking into account the collisional excitation from the He I $2^{3} \mathrm{~S}$ level, amounts to 0.160 , while Monk et al. (1988), that considered the collisional excitation, for the same object obtained a value of 0.118 .

The $\mathrm{He}^{+} / \mathrm{H}^{+}$ratio can be derived from different He I lines. For Type I $\mathrm{PNe}$ that show high electron densities and high electron temperatures, to 


\section{PLANETARY NEBULAE OF TYPE I REVISITED}

obtain agreement among the $\mathrm{He} / \mathrm{H}$ values derived from different $\mathrm{He} \mathrm{I}$ lines it is necessary to assume the presence of strong temperature fluctuations (Peimbert 1995, and references therein; Peña et al. 1995; Peimbert et al. 1995a).

The $\mathrm{C}^{++} / \mathrm{O}^{++}$values derived from the C II 4267 to [O III] 5007 intensity ratios and $T\left(\mathrm{C}^{++}\right)$for $36 \mathrm{PNe}$ of Types I and II are on average a factor of four higher than those derived from the C III] $1906+1909$ to [O III] 5007 intensity ratios and $T\left(\mathrm{O}^{++}\right)$, the difference is due to spatial temperature fluctuations and the first set of values should be preferred to the second one (Peimbert et al. 1995b). Moreover Peimbert et al. (1995a) based on the C II 4267 line intensities and $T\left(\mathrm{C}^{++}\right)$find that a group of $16 \mathrm{PNe}$ of Type I show $\mathrm{C} / \mathrm{O}>1$, implying that the the third dredge up has taken place in all of them.

To derive the $\mathrm{Ne} / \mathrm{O}$ value the following equation is often used

$$
\mathrm{Ne} / \mathrm{O}=\mathrm{Ne}^{++} / \mathrm{O}^{++}
$$

(e. g., Peimbert et al. 1992). In Figure 1 it can be seen that $\mathrm{Ne}^{++} / \mathrm{O}^{++}$ increases with decreasing density, indicating that for objects with $N_{e}<$ $2500 \mathrm{~cm}^{-3}$ equation (1) is a poor approximation to the $\mathrm{Ne} / \mathrm{O}$ value; this result probably is due to the presence of the charge exchange reaction $\mathrm{O}^{++}$ $+\mathrm{H}^{0} \rightarrow \mathrm{O}^{+}+\mathrm{H}^{+}$, that permits the coexistence of $\mathrm{Ne}^{++}$with $\mathrm{O}^{+}$(e. g., Pequignot 1980, and references therein). Ionization structure models predict that the lower the density the higher the $\mathrm{H}^{0} / \mathrm{H}^{+}$ratio consistent with the charge exchange suggestion and Figure 1, the true $\mathrm{Ne} / \mathrm{O}$ ratio is that derived from PNe with $N_{e}>2500 \mathrm{~cm}^{-3}$, and amounts to $-0.60 \pm 0.06 \mathrm{dex}$, in excellent agreement with the result derived from Type II PNe (TorresPeimbert \& Peimbert 1977; Peimbert 1990; Kingsburgh \& Barlow 1994), and implies that galacticType I PNe are not Ne rich. The values derived from Type I PNe with $N_{e}<2500 \mathrm{~cm}^{-3}$ are only upper limits to the real $\mathrm{Ne} / \mathrm{O}$ abundance. It has been noted that Type I PNe and bipolar PNe are Ne rich (Barlow 1991, and references therein; Corradi \& Schwarz 1995), this result has to be reanalized to see if it is due to objects with $N_{e}<2500$ $\mathrm{cm}^{-3}$.

To derive the $\mathrm{N} / \mathrm{O}$ value the following equation is often used

$$
\mathrm{N} / \mathrm{O}=\mathrm{N}^{+} / \mathrm{O}^{+},
$$

in the presence of the $\mathrm{O}$ charge exchange reaction some $\mathrm{O}^{+}$coexists with $\mathrm{O}^{++}$and with $\mathrm{N}^{++}$, and consequently equation (2) provides a lower limit to the $\mathrm{N} / \mathrm{O}$ value.

Some authors have found that there is a weak $\mathrm{O} / \mathrm{H}$ versus $\mathrm{N} / \mathrm{O}$ anticorrelation in galactic and extragalactic Type I PNe (Peimbert \& TorresPeimbert 1983, 1987; Aller et al. 1987; Peimbert 1985, 1990; Henry 1989, 


\section{S. Torres-Peimbert}

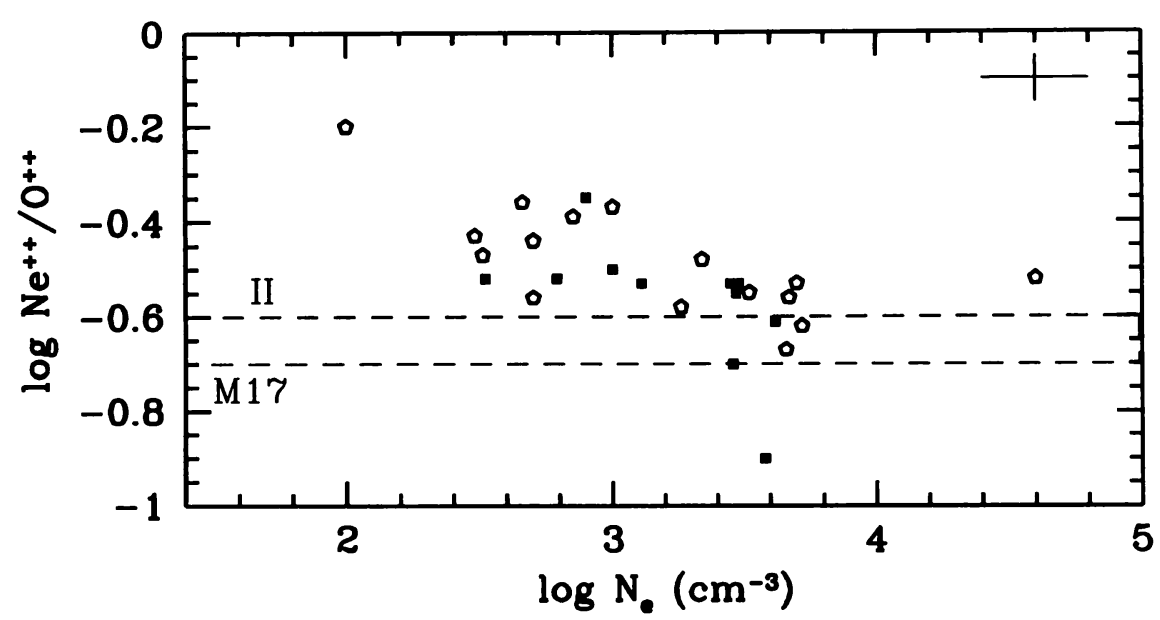

Figure 1. $\mathrm{Ne}^{++} / \mathrm{O}^{++}$values for galactic PNe of Type I; squares from Kingsburgh \& Barlow (1994), open symbols Peimbert et al. (1995a). The horizontal lines represent the values for Type II PNe and for M17, a galactic H II region (Peimbert et al. 1992). Notice that for $\log N_{e}<3.4 \mathrm{~cm}^{-3}$ all the objects have $\mathrm{Ne}^{++} / \mathrm{O}^{++}$values higher than -0.60 dex. (See also Faúndez-Abans et al. 1996.)

1990; Kaler et al. 1990; Dopita \& Meatheringham 1991; Jacoby \& Kaler 1993; Leisy \& Dennefeld 1996), while others have not found it (Kingsburgh \& Barlow 1994). Two possible explanations have been advanced in the literature to explain this trend: ON cycling and spatial temperature fluctuations. Peimbert et al. (1995a) find that for galactic PNe the trend is due to temperature fluctuations and not to $\mathrm{ON}$ cycling; they find that lower $\mathrm{N} / \mathrm{O}$ and higher $\mathrm{O} / \mathrm{H}$ ratios are obtained if $T\left(\mathrm{C}^{++}\right)$is used instead of $T\left(\mathrm{O}^{++}\right)$. Moreover they find also that the $\mathrm{Ar} / \mathrm{O}$ ratio in Type I PNe is practically the same as that derived from $\mathrm{H}$ II regions of the solar neighborhood; in the presence of $\mathrm{ON}$ cycling we would have expected an excess in the Ar/O ratio since $\mathrm{Ar}$ is not affected by the evolution of intermediate mass stars.

Dopita \& Meatheringham (1991) find that Type I PNe in the MC show excesses of $\mathrm{Ne}, \mathrm{Ar}$, and $\mathrm{S}$ relative to $\mathrm{O}$ and suggest that they are due to ON cycling. It is also possible that spatial temperature fluctuations and the breakdown of equation (1) are partially responsible for this result. 


\section{PLANETARY NEBULAE OF TYPE I REVISITED}

\section{Some open questions}

Why are Type I PNe bipolar? Calvet \& Peimbert (1983) proposed that their bipolar nature could be a direct consequence of the large masses and angular momenta of their progenitor stars, García-Segura et al. (1996) and Mellema (1996) have produced models in agreement with this suggestion. It has also been proposed that the bipolar nature is due to binary evolution (e. g., Livio 1993; Iben 1993, and references therein). Maybe some bipolar nebulae are produced by single stars and others by a binary mechanism (two stars or one star and a planet).

Are all bipolars Type I PNe? Of a galactic group of 51 bipolar PNe with abundance determinations 45 are of Type I (Torres-Peimbert \& Peimbert 1996). Are all Type I PNe bipolars? Of a galactic group of 54 Type I PNe with morphology 45 are bipolars (Torres-Peimbert \& Peimbert 1996). The discrepant numbers could be due to errors in the abundance determinations or in the morphological classification, or to real physical differences.

Can PNe of Type I be subdivided based on physical characteristics? Faúndez-Abans et al. (1996) have suggested that they can be divided in three groups according to their $\mathrm{C} / \mathrm{O}$ and $\mathrm{He} / \mathrm{H}$ ratios. Leisy \& Dennefeld (1996) have suggested that PNe of type I in the MC do not form a homogeneous group.

It is beyond the scope of this review to discuss thoroughly the answers to these questions.

It is a pleasure to acknowledge fruitful discussions with G. GarcíaSegura and M. Peña.

\section{References}

Acker, A. 1980, A\&A, 89, 33.

Acker, A. 1983, in IAU Symposium 103, Planetary Nebulae, ed. R.D. Flower (Dordrecht: Reidel), 241.

Aller, L.H., Keyes, C.D., Maran, S.P., Gull, T.R., Michalitsianos, A.G. \& Stecher, T.P. 1987, ApJ, 320, 159.

Balick, B. 1987, AJ, 94, 671.

Balick, B. 1989, in IAU Symposium 131, Planetary Nebulae, ed. S. Torres-Peimbert (Dordrecht: Reidel), 83.

Barlow, M.J. 1991, in IAU Symposium 148, The Magellanic Clouds, eds. R. Haynes \& D. Milne (Dordrecht: Kluwer), 291

Calvet, N. \& Peimbert, M. 1983, RevMexAA, 5, 319.

Carigi, L. 1994, ApJ, 424, 181.

Cazetta, J.O. \& Maciel, W.J. 1994, A\&A, 290, 936.

Corradi, R.L.M. \& Schwarz, H. E. 1995, A\&A, 293, 871.

Cudworth, K.M. 1974, AJ, 79, 1384.

Dopita, M.A. 1993, in IAU Symposium 155, Planetary Nebulae, eds. R. Weinberger \& A. Acker (Dordrecht: Kluwer), 433.

Dopita, M.A. \& Meatheringham, S.J. 1991, ApJ, 377, 480.

Dopita, M.A. \& Sutherland, R.S. 1996, ApJS, 102, 161. 


\section{S. Torres-Peimbert}

Dopita, M.A., Vassiliadis, E., Meatheringham, S.J., Bohlin, R.C., Ford, H.C., Harrington, J.P., Wood, P.R., Stecher, T.P. \& Maran, S.P. 1996, ApJ, 460, 320.

Dufour, R.J. 1984, in IAU Symposium 108, Structure and Evolution of the Magellanic Clouds, eds. S. van den Bergh \& K. de Boer (Dordrecht: Reidel), 353.

Dutra, C.M. \& Maciel, W.J. 1990, RevMexAA, 21, 264.

Faúndez-Abans, M., de Oliveira-Abans, M., \& Ormeño, M.I. 1996, A\&A, 306, L1.

García-Segura, G., Langer, N., Różyczka, M., Mac Low, M.-M. \& Franco, J. 1996, these proceedings.

Garnett, D.R., Skillman, E.D., Dufour, R.J., Peimbert, M., Torres-Peimbert, S., Terlevich, R., Terlevich, E. \& Shields, G.A. 1995, ApJ, 443, 64.

Gathier, R. \& Pottasch, S.R. 1985, Production and Distribution of C, N, O Elements, eds. I.J. Danziger et al. (ESO), 307.

Gómez, Y., Moran, J.M., Rodríguez, L.F. \& Garay, G. 1989, ApJ, 345, 862.

Gorny, S.K., Stasinska, G. \& Tylenda, R. 1996, A\&A, in press.

Greig, W.E. 1971, A\&A, 10, 161.

Greig, W.E. 1972, A\&A, 18, 70.

Henry, R.B.C. 1989, MNRAS, 241, 453.

Henry, R.B.C. 1990, ApJ, 356, 229.

Huggins, P.J. 1993, in IAU Symposium 155, Planetary Nebulae, ed. R. Weinberger \& A. Acker (Dordrecht: Kluwer), 147.

Huggins, P.J., Bachiller, B., Cox, P. \& Forveille, T. 1996, A\&A, submitted.

Iben, I. Jr. 1993, in IAU Symposium 155, Planetary Nebulae, eds. R. Weinberger \& A. Acker (Dordrecht: Kluwer), 587.

Jacoby, G.H. \& Kaler, J.B. 1993, 417, 209.

Kaler, J.B. 1983, in IAU Symposium 103, Planetary Nebulae, ed. R.D. Flower (Dordrecht: Reidel), 245.

Kaler, J.B. \& Jacoby, G.H. 1989, ApJ, 345, 871.

Kaler, J.B. \& Jacoby, G.H. 1990, ApJ, 362, 491.

Kaler, J.B., Shaw, R.A. \& Kwitter, K.B. 1990, Apj, 359, 392.

Kastner, J.H., Weintraub, D.A., Gatley, I., Merrill, K.M. \& Probst, R.G. 1996, ApJ, 462, 777.

Kingsburgh, R.L. \& Barlow, M.J. 1994, MNRAS, 271, 257.

Leisy, P. \& Dennefeld, M. 1996, A\&AS, 116, 95.

Livio, M. 1993, in IAU Symposium 155, Planetary Nebulae, eds. R. Weinberger \& A. Acker (Dordrecht: Kluwer), 279.

Maciel, J.W. \& Dutra, C.M. 1992, A\&A, 262, 271.

Mellema, G. 1996, these proceedings.

Monk, D.J., Barlow, M.J. \& Clegg, R.E.S. 1988, MNRAS, 234, 583.

Pasquali, A. \& Perinotto, M. 1993, A\&A, 280, 581.

Peimbert, M. 1978, in IAU Symposium 76, Planetary Nebulae, ed. Y. Terzian (Dordrecht: Reidel), 215.

Peimbert, M. 1985, RevMexAA, 10, 125.

Peimbert, M. 1990, Rep. Prog. Phys, 53, 1559.

Peimbert, M. 1995, in The Analysis of Emission Lines, eds. R.E. Williams \& M. Livio (Cambridge: Cambridge Univ. Press), 168.

Peimbert, M., Luridiana, V. \& Torres-Peimbert, S. 1995a, RevMexAA, 31, 147.

Peimbert, M. \& Torres-Peimbert, S. 1983, in IAU Symposium 103, Planetary Nebulae, ed. R.D. Flower (Dordrecht: Reidel), 233.

Peimbert, M. \& Torres-Peimbert, S. 1987, RevMexAA, 14, 540.

Peimbert, M., Torres-Peimbert, S. \& Luridiana, V. 1995b RevMexAA, 31, 131.

Peimbert, M., Torres-Peimbert, S. \& Ruiz, M.T. 1992 RevMexAA, 24, 155.

Peña, M., Peimbert, M., Torres-Peimbert, S., Ruiz, M.T. \& Maza, J. 1995, ApJ,441, 343.

Pequignot, D. 1980, A\&A, 81, 356.

Pottasch, S. 1989, in IAU Symposium 131, Planetary Nebulae, ed. S. Torres-Peimbert (Dordrecht: Reidel), 481. 


\section{PLANETARY NEBULAE OF TYPE I REVISITED}

Rola, C. \& Stasinska, G. 1994, A\&A, 282, 199.

Sabbadin, F. 1986, A\&A, 160, 31.

Stanghellini, L., Corradi, R.L.M. \& Schwarz, H.E. 1993, A\&A, 279, 521.

Stasinska, G. \& Tylenda, R. 1990, A\&A, 240, 467.

Torres-Peimbert, S. \& Peimbert, M. 1977, RevMexAA, 2, 181.

Torres-Peimbert, S. \& Peimbert, M. 1996, in preparation

Walton, N.A., Barlow, M.J., Clegg, R.E.S. \& Monk, D.J. 1991, in IAU Symposium 148,

The Magellanic Clouds, eds. R. Haynes \& D. Milne (Dordrecht: Kluwer), 334

Webster, B.L., Payne, P.W., Storey, J.W.V. \& Dopita, M.A. 1988, MNRAS, 235, 533.

Zuckerman, B. \& Gatley, I. 1988, ApJ, 324, 501. 